Central Washington University

ScholarWorks@CWU

All Faculty Scholarship for the College of the Sciences

7-1997

\title{
Metastable states in classical and quantum systems
}

\author{
Mark Makela \\ Central Washington University \\ Samantha Parmley \\ Central Washington University \\ Roger Yu \\ Central Washington University
}

Follow this and additional works at: https://digitalcommons.cwu.edu/cotsfac

Part of the Quantum Physics Commons, and the Science and Mathematics Education Commons

\section{Recommended Citation}

Makela, M., Parmley, S., \& Yu, R. (1997). Metastable states in classical and quantum systems. American Journal of Physics, 65(7), 653-657. https://doi.org/10.1119/1.18622

This Article is brought to you for free and open access by the College of the Sciences at ScholarWorks@CWU. It has been accepted for inclusion in All Faculty Scholarship for the College of the Sciences by an authorized administrator of ScholarWorks@CWU. For more information, please contact scholarworks@cwu.edu. 
Metastable states in classical and quantum systems

\author{
Mark Makela, Samantha Parmley, and Roger Yu \\ Department of Physics, Central Washington University, Ellensburg, Washington 98926
}

(Received 6 May 1996; accepted 14 January 1997)

\begin{abstract}
The classical vibrational metastable states in a one-dimensional two-mass system are investigated experimentally and theoretically via the transmission of a coherent wave packet propagating through the system. The Fourier transform of the vibrational signal recorded in between the masses reveals resonant excitations by the coherent wave packet. The time-resolved spectrum indicates that the lifetime of a metastable state of higher frequency is longer than that of lower frequency, which is in contrast with the quantum mechanical double-barrier system. This study, which is easily accessible to physics majors, also demonstrates quantum resonant tunneling in a very simple classical way. (C) 1997 American Association of Physics Teachers.
\end{abstract}

The concept of metastable states in quantum mechanics dates back to the beginning of the century. The interaction between a quantum system and an external electromagnetic field was described by Planck using the concept of a metastable state. A metastable state is an unstable and exponentially decaying state with a certain lifetime. It is distinguished from a stationary state, which is "infinitely" long lived. For the sake of simplicity, we discuss the onedimensional quantum scattering problem: ${ }^{1}$ the description of the metastable states and the transmission coefficient of a plane wave. Figure 1(a) illustrates a schematic diagram of the double-barrier structure under consideration. The stationary quantum scattering problem is governed by the timeindependent Schrödinger equation

$$
\left[-\frac{\hbar^{2}}{2 m} \frac{d^{2}}{d x^{2}}+V(x)\right] \Psi=E \Psi,
$$

where $m$ is the mass of an electron and $V(x)=\alpha \delta(x)$ $+\alpha \delta(x-d)$ is the potential energy of the electron. The constants $\alpha$ and $d$ are the barrier strength and barrier separation. Some of the eigenstates are mainly confined in between the barriers, and their energies form a discrete spectrum. The discrete eigenstates are metastable because their energies are above a stable ground state taken to be at $E=0$. The instability of such a state, i.e., the decay of an electron from the $n$th metastable state in the potential well, can be described by a complex energy, ${ }^{2}$

$$
\bar{E}_{n}=E_{n}+i \Gamma_{n} / 2 \text {. }
$$

With this complex variable, the time-dependent part of the wave function for the $n$th state has the following form:

$$
\Psi(t) \propto \exp \left[-t \Gamma_{n} / 2 \hbar\right] \exp \left[-i E_{n} t / \hbar\right] .
$$

The physical quantity $\hbar / \Gamma_{n}$ then, is the lifetime of the $n$th metastable state. The energy spectrum of the system when electrons escape from the $n$th unstable state is obtained by the Fourier transform

$$
\begin{aligned}
S(E) & \propto \int_{0}^{\infty} \exp \left[-t \Gamma_{n} /(2 \hbar)-i t\left(E-E_{n}\right) / \hbar\right] d t \\
& =\frac{\hbar}{i\left(E-E_{n}\right)-\Gamma_{n} / 2} .
\end{aligned}
$$

The probability density per unit energy of finding the energy $E$ for the emitted electron from the state $n$ is proportional to the square of $S(E)$ :

$$
|S(E)|^{2}=\frac{\hbar^{2}}{\left(E-E_{n}\right)^{2}+\left(\Gamma_{n} / 2\right)^{2}} .
$$

This function peaks at $E=E_{n}$. The characteristic width $\Delta E_{n}$ broadens as one moves to a higher unstable state, because of the stronger overlap between the wave functions inside and outside the double-barrier structure. Accordingly, the lifetime of a higher metastable state shortens. The lifetime of a metastable electronic state can also be determined by the transmission coefficient of a harmonic plane wave tunneling through the double-barrier structure. ${ }^{3}$ By solving the Schrödinger equation with the double delta barrier potential, as illustrated in Fig. 1(a), and using the standard boundary conditions at the barriers, one finds the transmission coefficient of an electronic harmonic wave (see the Appendix):

$$
T=|\tau|^{2}
$$

where

$$
\tau=4 /\left[\epsilon^{2} e^{2 i k d}+(i \epsilon+2)^{2}\right] .
$$

Here, the dimensionless parameter $\epsilon$ is $2 \alpha m /\left(\hbar^{2} k\right)$, and the wave vector $k$ equals $\left(2 m E / \hbar^{2}\right)^{1 / 2}$. We have plotted the absolute value of $\tau$ vs energy $E$ in Fig. 1(a) by using specific values of $d(=100 \AA), \alpha\left(=2.0 \mathrm{eV} \AA\right.$ ), and $m_{e}$ (electron mass). The resonant peaks corresponding to the metastable states become broadened at higher energy indicating the decreasing lifetime of the metastable states.

This quantum mechanical phenomenon has a strong analogy in classical systems and it is our intention to demonstrate it in this article. We first consider theoretically an infinitely long string loaded with two equal masses separated by distance $d$ as shown in Fig. 1(b). Classically, the two masses restrict the interaction between the string segments inside and outside, leading to a resonant frequency spectrum for the string segment confined by the masses. The quantized states are metastable and decay exponentially like the metastable states in the quantum double-barrier system. The resonant states confined between the masses would be stable or stationary if the masses were infinitely large. The waveforms of the metastable states are similar to the standing waves found in a clamped bare string of length $d$. 

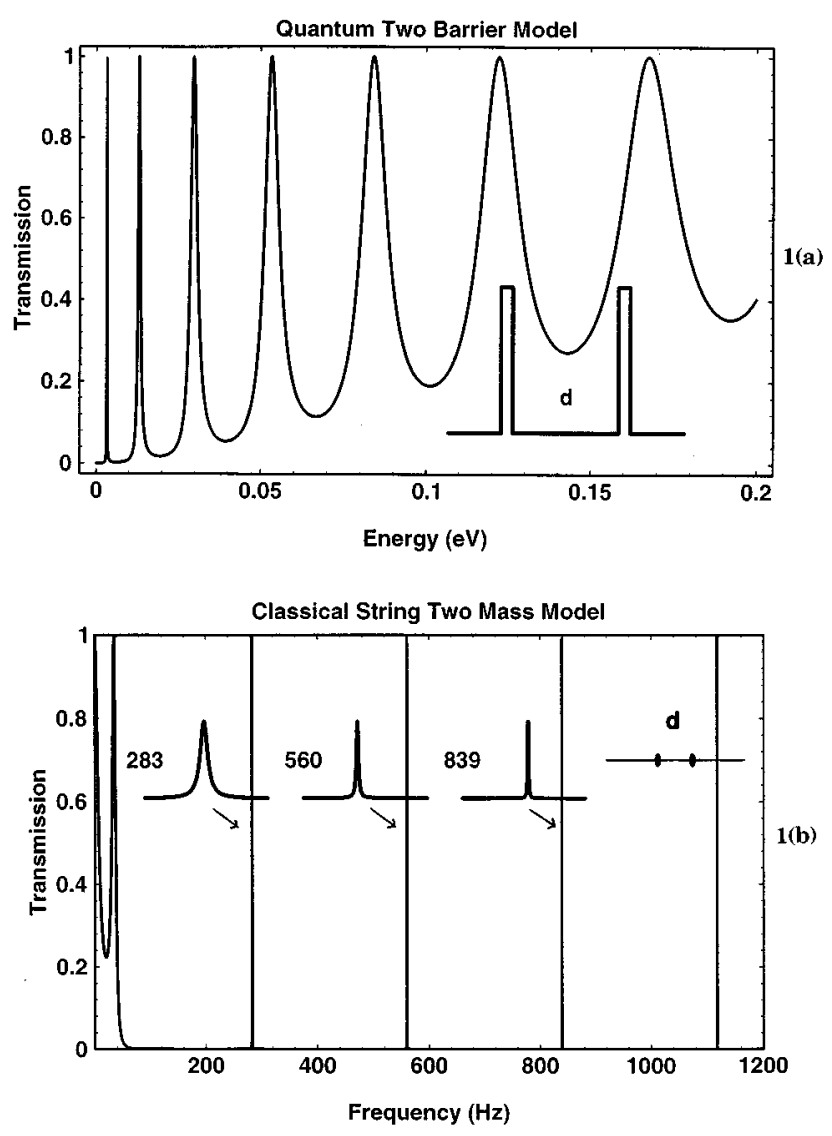

Fig. 1. The calculated transmission amplitude $|\tau|$ calculated using the twodelta-barrier model. For the classical system the parameters used in the calculation are matched with the experiment: $T_{0}=40.7 \mathrm{~N}, m=5.0 \mathrm{~g}, \sigma$ $=1.2 \times 10^{-3} \mathrm{~kg} / \mathrm{m}$, mass separation $d=0.33 \mathrm{~m}$ and total length of the string $L=6.5 \mathrm{~m}$. In order to show the resonant width, the peaks at 283,560 , and $839 \mathrm{~Hz}$ are widened by a factor of 60 and displayed by the inserts. The frequency intervals for the three peaks are from 282 to $285 \mathrm{~Hz}, 559$ to 562 $\mathrm{Hz}$ and from 837 to $840 \mathrm{~Hz}$. For the quantum system, $d=100 \AA$, $\alpha$ $=2.0 \mathrm{eV} \AA, m=m_{e}$.

Despite the strong analogy between the classical and quantum metastable states, there is a crucial difference between them: The lifetime of a higher frequency metastable state in the classical two-mass system becomes longer. We have also calculated the transmission coefficient $T$ of a classical plane wave through the two-mass system as a function of the harmonic frequency by solving the time-independent wave equation ${ }^{4}$

$$
T_{0} \ddot{u}(x)+\sigma \omega^{2} u(x)=0,
$$

where, $T_{0}$ is the tension, $\sigma(x)=\sigma+m \delta(x)+m \delta(x-d)$ is the mass density, $u(x)$ is the transverse displacement of the string, $\omega$ is the harmonic frequency, and $d$ is the mass separation. The boundary condition matching at the locations of the masses results in a transmission coefficient which appears similar to the quantum counterpart [Eq. (6)] (see the Appendix), except that the dimensionless parameter $\epsilon$ now equals $m \omega /\left(T_{0} \sigma\right)^{1 / 2}$. Figure 1(b) depicts the absolute value of the transmission amplitude $\left(=T^{1 / 2}\right)$ plotted against the frequency. The resonant peaks, shown by the inserts, sharpen at higher frequencies; therefore, the lifetime becomes longer. Actually, in the limit of large $\epsilon$, the lifetimes of both systems can be approximated analytically. Here, the argu- ment is presented in a parallel fashion. Start with the dimensionless parameter $\epsilon$ : $\epsilon_{Q}=2 \alpha m / \hbar^{2} k$, and $\epsilon_{C}=\omega m / \sqrt{T_{0} \sigma}$ $=(m / \sigma d) \omega d / v$. It follows from Eq. (6) that for large $\epsilon$, $|\tau| \approx 2 /\left(\epsilon^{2} \sin (k d)\right) ;|\tau|$ is a maximum at $k d=n \pi$ as expected. To find the width of the resonant peaks, let $|\tau|$ $\approx 2 /\left(\epsilon^{2} \sin (k d)\right)=1 / 2$, then $\sin k d= \pm 4 / \epsilon^{2} \ll 1$, and $k d$ $=n \pi \pm 4 / \epsilon^{2}$, so $\Delta k=8 /\left(\epsilon^{2} d\right)$. Due to the different dispersion relations, $E=\hbar^{2} k^{2} / 2 m$, and $\omega=v k$, we found $\Delta E$ $\sim E^{3 / 2}$, and $\Delta \omega \sim \omega^{-2}$. The uncertainty principle yields the quantum lifetime $\sim E^{-3 / 2}$ and the classical lifetime $\sim \omega^{2}$. The physical interpretation of longer lifetime for resonant state of higher frequency in the classical system is quite clear. At lower frequencies, the wavelength is longer than the mass separation so the propagating wave "views" the two masses as one. As the wavelength decreases, the size of the mass separation becomes much more critical in confining the metastable states.

The classical metastable states can be excited by sending a propagating mechanical wave packet through the two-mass system. The string segment between the masses responds to the incident wave packet strongly if the mean wavelength of the incident wave and that of the metastable states become close. In general the incident wave packet contains many plane waves of different wavelengths so that several metastable states may be excited. Because the resonant transmission peaks are so sharp, the two masses actually serve as a filter. As the wave packet impinges on the masses, some of the harmonic waves are reflected, and the waves whose frequencies match those of the metastable states are transmitted. The part of the wave packet that can tunnel through the masses contains the harmonic components of frequencies of the metastable states. In the experimental setup, similar to the one we used in studying the eigenstates of a loaded string, ${ }^{5}$ two 5 -g masses separated by $0.33 \mathrm{~m}(=d)$ were attached at the middle of a $6.5-\mathrm{m}(=L)$ long brass harpsichord wire. The wire with linear mass density $\sigma=1.2 \times 10^{-3} \mathrm{~kg} / \mathrm{m}$, was tensioned to $40.7 \mathrm{~N}$. Pulses of full width at halfmaximum (FWHM) of about $0.33 \mathrm{~m}$ were delivered to the wire via an audio speaker with an aluminum rod attached to the voice coil. The speaker was pulsed with a trianglelike wave derived from a Tektronix function generator and a 200-W amplifier. Figure 2(a) depicts the initial pulse and its spectral distribution. As the pulse propagates through the string, a transducer records the ac signal and runs it through the linear amplifier into the computer driven digitizer. The digitized data enable us to analyze the real time series in the frequency domain by fast Fourier transform (FFT). Figure 2(b) shows the short-period $(\sim 0.02-\mathrm{s})$ transmitted signal recorded on the far side of the masses and its Fourier transform. At the early stage of the transmission, the signal is dominated by the vibration of about $50 \mathrm{~Hz}$, modulated by modes near 300 and $600 \mathrm{~Hz}$. Figure 2(c) displays the Fourier transform of the long-period time series of a few seconds, recorded in between the masses: more peaks and structure are shown in the figure. In order to fully understand the experimental data, one needs to solve the eigenequation [Eq. (7)], in conjunction with the boundary conditions, $u(0)$ $=u(L)=0$. Very recently we have developed a method of solving the finite-length $[L=6.5(\mathrm{~m})]$ problem based on sine-wave expansion. ${ }^{5}$ For the present finite-length problem, the mass density in Eq. (7) is expressed as $\sigma(x)=\sigma$ $+m \delta(x-(L / 2-d / 2))+m \delta(x-(L / 2+d / 2))$. The method 
a)
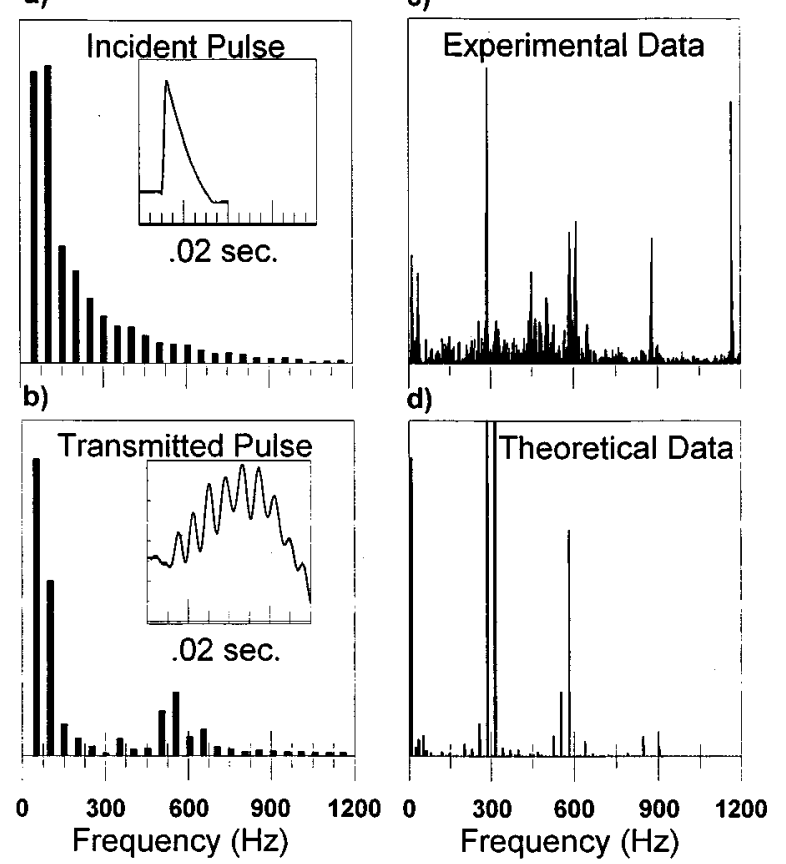

Fig. 2. (a) and (b), respectively, display the experimental incident and transmitted wave shapes and their Fourier components; (c) and (d) show the experimental and theoretical density of states in between the masses. Arbitrary units are used for all the vertical axes.

was adapted in this study and the eigenfunctions $u_{n}(x)$ and eigenfrequencies $\omega_{n}$ are obtained accurately. The dynamics of the loaded string can be expressed in terms of the eigenstates by the superposition principle:

$$
|u(x, t)\rangle=\sum_{n=1}^{\infty}\left|u_{n}(x)\right\rangle d_{n} \cos \left(\omega_{n} t\right) .
$$

The density of states, $d_{n}=\left\langle u_{n}(x) \mid u(x, o)\right\rangle$, is determined by the initial displacement of the string. The Fourier transform of Eq. (8) gives rise to the spectrum at a certain position,

$$
s(x, \omega)=\sum_{m} d_{m} u_{m}(x) \delta\left(\omega-\omega_{m}\right) .
$$

In the above equation, $d_{m} u_{m}(x)$ is the local density of states which reveals the information about the density of harmonic components at position $x$. If $x$ is in between the masses, the local spectrum $S(x, \omega)$ is expected to peak at the frequencies of metastable states. Figure 2(d) displays the theoretical local spectrum $(x=L / 2+d / 4)$ calculated using long-period time series, so it is directly comparable to Fig. 2(c). The wave speed in the string is approximately equal to $\left(T_{0} / \sigma\right)^{1 / 2}$ $=180 \mathrm{~m} / \mathrm{s}$, the wavelength of the lowest metastable state is $\lambda_{1}=2 d=0.66 \mathrm{~m}$, and the frequency of the state is about 290 $\mathrm{Hz}$. The frequency of the metastable states jumps upwards in steps of $290 \mathrm{~Hz}$. Because of the finite length of the entire string, the frequency spectrum of the entire system is also discrete: The frequency separation is roughly equal to $180 \mathrm{~m} / \mathrm{s} /(2 \times 6.5 \mathrm{~m})=14 \mathrm{~Hz}$. By observing Figs. 2(c) and 2(d), one can identify four kinds of resonant excitations as the wave packet tunnels through the double-mass system: (a) metastable resonances near 290, 580, 870, and $1160 \mathrm{~Hz}$; (b) discrete background structure, peaks separated by about 14
$\mathrm{Hz}$ corresponding to the resonances of the entire string; (c) strong peaks at $18 \mathrm{~Hz}$ due to the fundamental resonant mode of the entire string; (d) the well-defined peak at about $50 \mathrm{~Hz}$ which can be attributed to the rotational vibration of the two masses. The calculated eigenfunction at $50 \mathrm{~Hz}$ (not shown here) displays large out-of-phase displacement of the masses and a straight line in between them: It is a strong indication of dumbbell-like rotational motion. Because of the spatial resolution of the pickup (the size of the pickup is about 10 $\mathrm{cm}$ ), the metastable states beyond $1200 \mathrm{~Hz}$, whose wavelengths are shorter than $7 \mathrm{~cm}$, were not detected. The calculated spectrum misses the mode at $1160 \mathrm{~Hz}$ because the chosen position $(x=L / 2+d / 4)$ is right on a node of the mode. The string segment in between the masses couples with the string outside through the masses. This interaction is responsible for the frequency splitting shown in Figs. 2(c) and 2(d) near 300 and $600 \mathrm{~Hz}$. The short-period transmitted vibrational signal and its Fourier transform [Fig. 2(b)] does not contain the $18 \mathrm{~Hz}$ mode because the corresponding period is longer than $0.02 \mathrm{~s}$. The higher metastable states of 870 and $1160 \mathrm{~Hz}$ do not play a significant role in the early stage of the transmission because of their longer lifetime: They take longer to radiate the energy out of the region confined by the masses.

The experimental spectrum shown in Fig. 2(c) is the Fourier transform of this entire time series of $1.0 \mathrm{~s}$. It tells us how many metastable states are excited over the period but it does not provide any indication of how long the metastable state of a certain frequency lasts. We have used the shortperiod Fourier transform to study the lifetime of metastable states: We break up the time series into small segments and Fourier analyze each time segment. ${ }^{6}$ We shall call the timedependent spectrum the spectrogram. The time series was divided into 32 pieces to obtain the spectrogram. The spectrograms of frequencies near the lowest four metastable states are plotted semilogarithmically in Fig. 3. At lower frequencies [Figs. 3(a) and 3(b)], the coupling between the metastable states and the rest of the system is much stronger, as shown in Figs. 2(c) and 2(d) by the mode splitting, so the spectrograms display oscillations. The spectrograms of 859 and $1171 \mathrm{~Hz}$ basically follow straight lines, indicating the nature of exponential decay. The slopes of the straight lines are proportional to the reciprocal of the lifetime of the states. At high frequencies, the slope is slightly smaller so the lifetime is longer. The lifetimes for the lowest four metastable states are found to be approximately $0.2,0.3,0.4$, and $0.4 \mathrm{~s}$, respectively, by finding the slopes in Fig. 3. One can also estimate the lifetime of a metastable state using the analytical equations derived above. For the present experimental parameters $\left(T_{0}=40.7 \mathrm{~N}, m=5.0 \mathrm{~g}, \sigma=1.2 \times 10^{-3} \mathrm{~kg} / \mathrm{m}\right.$, mass separation $d=0.33 \mathrm{~m}), \epsilon$ equals $\omega m /\left(T_{0} \sigma\right)^{1 / 2} \sim 0.023 \omega$, and $\Delta k$ equals $8 /\left(d \epsilon^{2}\right)-4.6 \times 10^{4} / \omega^{2}$. The resonant width $\Delta \omega$ is equal to $v \Delta k \sim 8.3 \times 10^{6} / \omega^{2}$. Because of the uncertainty relation, $\Delta \omega \Delta t \sim 2 \pi$, the lifetime $\Delta t$ equals $2 \pi \omega^{2} /(8.3$ $\left.\times 10^{6}\right)$. If the fundamental frequency is used $(\omega=2 \pi \times 290$ $\mathrm{Hz}$ ), the lifetime is about $2.6 \mathrm{~s}$ whose inverse is quite close to the width of the resonant peak shown in Fig. 1(b) near 290 $\mathrm{Hz}$. However, the lifetime is an order of magnitude larger than the experimental data. We believe that the broadening of the experimental resonant peaks can be attributed to the interaction between the masses and the string. The string segment in between the masses loses energy to the masses, which serve as two dampers, and therefore broadens the resonant peak. 


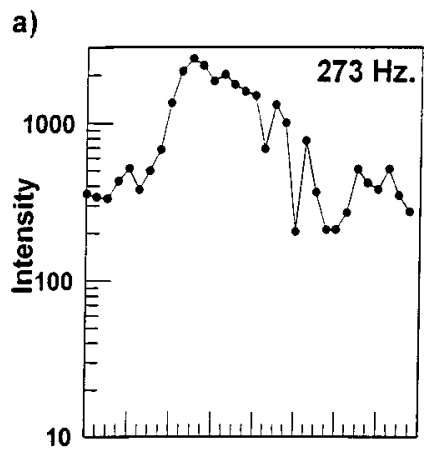

b)

c)
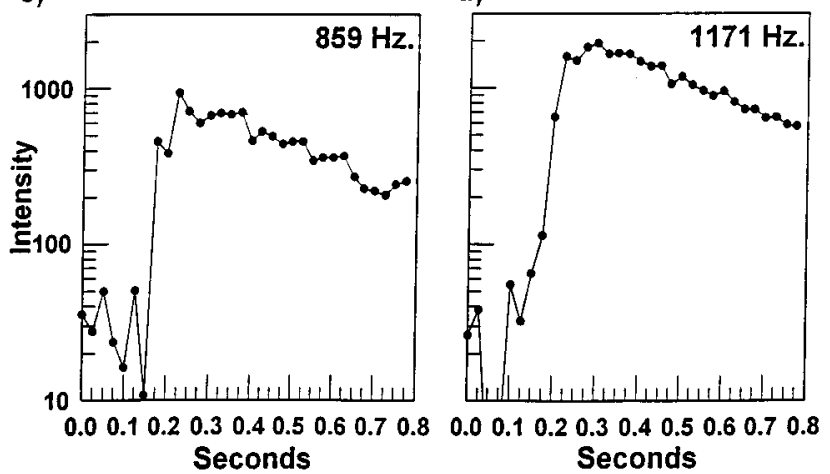

Fig. 3. Four spectrograms at the lowest four resonant frequencies are plotted in arbitrary units.

In conclusion, we have studied the resonant tunneling and metastable states in a classical one-dimensional two-mass system using a coherent wave packet. The excited metastable states confined in between the masses decay exponentially. The transmission of the wave packet happens as the excited metastable states radiate their energy. We have found that the lifetime of a metastable state with higher frequency is longer than that of lower-frequency modes, which is in contrast with the quantum-mechanical result. We have also found an interesting dumbbell-like rotational vibration of the masses. The eigenproperties of the classical system are determined accurately using our newly developed sine-wave expansion method. The dynamics of the wave packet is described by superimposing the eigenstates. The experimental and theoretical resonant peak locations agree with each other very well. Some experimental evidence has been found indicating that the lifetimes of metastable states in the string-mass system at higher frequency are longer. This investigation may provide some insight into the dynamics of coherent states in a solid-state multiple-quantum-well system. The experiment and the theoretical approach are also accessible to physics majors. The experiment has been integrated into our quantum mechanics course as a supplemental project.

\section{ACKNOWLEDGMENTS}

One of the authors (SP) appreciates the support from the Council for Undergraduate Research Summer Opportunities for Research (CURSOR) Fellowship. R. Y. appreciates the financial support financial support from the College of Graduate Studies and Research at Central Washington University and thanks Dr. Willard Sperry for many fruitful discussions.

\section{APPENDIX}

To find the transmission coefficient of a harmonic wave, we start with the time-independent Schrödinger equation:

$$
\left[-\frac{\hbar^{2}}{2 m} \frac{d^{2}}{d x^{2}}+V(x)\right] \Psi=E \Psi,
$$

where $V(x)=\alpha \delta(x)+\alpha \delta(x-d)$. The solution in three different regions can be expressed as follows:

$$
\begin{aligned}
& \Psi_{\mathrm{I}}(x)=e^{i k x}+\mathrm{Re}^{-i k x}, \quad x \leqslant 0, \\
& \Psi_{\mathrm{II}}(x)=A e^{i k x}+B e^{-i k x}, \quad 0 \leqslant x \leqslant d, \\
& \Psi_{\mathrm{III}}(x)=\tau e^{i k x}, \quad x \geqslant d .
\end{aligned}
$$

Here, $R$ and $\tau$ are the reflection and transmission amplitudes, respectively, and $k=\left(2 m E / \hbar^{2}\right)^{1 / 2}$. The transmission coefficient $T$ equals the square of the modulus of $\tau, T=|\tau|^{2}$. The boundary conditions at $x=0$ and $x=d$ are used to determine the amplitude $\tau$. At the locations of the delta barriers, the wave functions are continuous, that is

$$
\Psi_{\mathrm{I}}(0)=\Psi_{\mathrm{II}}(0), \quad \Psi_{\mathrm{II}}(d)=\Psi_{\mathrm{III}}(d) .
$$

The slope of the wave function at $x=0$ and $x=d$ is discontinuous, and it can be shown by carrying out the following integrals:

$$
\begin{aligned}
& \lim _{\epsilon \rightarrow 0} \int_{-\epsilon}^{+\epsilon}\left[-\frac{\hbar^{2}}{2 m} \frac{d^{2}}{d x^{2}}+\alpha \delta(x)+\alpha \delta(x-d)\right] \Psi(x) d x \\
& \quad=\lim _{\epsilon \rightarrow 0} \int_{\epsilon}^{+\epsilon} E \Psi(x) d x,
\end{aligned}
$$

so

$$
-\frac{\hbar^{2}}{2 m}\left[\Psi_{\mathrm{II}}^{\prime}(0)-\Psi_{\mathrm{I}}^{\prime}(0)\right]+\alpha \Psi_{\mathrm{I}}(0)=0
$$

and

$$
\begin{gathered}
\lim _{\epsilon \rightarrow 0} \int_{d-\epsilon}^{d+\epsilon}\left[-\frac{\hbar^{2}}{2 m} \frac{d^{2}}{d x^{2}}+\alpha \delta(x)+\alpha \delta(x-d)\right] \Psi(x) d x \\
\quad=\lim _{\epsilon \rightarrow 0} \int_{d-\epsilon}^{d+\epsilon} E \Psi(x) d x,
\end{gathered}
$$

or

$$
-\frac{\hbar^{2}}{2 m}\left[\Psi_{\mathrm{III}}^{\prime}(d)-\Psi_{\mathrm{II}}^{\prime}(d)\right]+\alpha \Psi_{\mathrm{III}}(d)=0 .
$$

From Eqs. (A4) and (A5), the discontinuity of the first-order derivatives of the wave functions at $x=0$, and $d$ can be observed. By substituting Eq. (A2) into Eqs. (A3), (A4), and (A5), we find

$$
\begin{gathered}
1+R=A+B-\frac{\hbar^{2}}{2 m}[A k i-B k i-k i+k R i]+\alpha(1+R) \\
=0, \\
A e^{i k d}+B e^{-i k d}=\tau e^{i k d}-\frac{\hbar^{2}}{2 m}\left[\tau k e^{i k d} i-A k e^{i k d} i\right. \\
\left.+B k e^{-i k d} i\right]+\alpha \tau e^{i k d}=0 .
\end{gathered}
$$


We find it easier to work with the dimensionless parameter $\epsilon$ which is defined as $\epsilon=2 \alpha m /\left(\hbar^{2} k\right)$. Equation (A6) now reads

$$
\begin{aligned}
& 1+R=A+B, \\
& A-B-1+R-\frac{\epsilon}{i}(1+R)=0, \\
& A e^{i k d}+B e^{-i k d}=\tau e^{i k d}, \\
& -A e^{i k d}+B e^{-i k d}+\left(1-\frac{\epsilon}{i}\right) \tau e^{i k d}=0 .
\end{aligned}
$$

Some simple substitutions result in the following quantum transmission amplitude;

$$
\tau=4 /\left[\epsilon^{2} e^{i k d}+(i \epsilon+2)^{2}\right] .
$$

For a classical string-two-mass system, the transmission amplitude can be found by using a similar method (see Ref. 4). The classical transmission amplitude is given by

$$
\tau=4 /\left[\epsilon^{2} e^{2 i k d}+(-i \epsilon+2)^{2}\right] .
$$

Here, the dimensionless parameter $\epsilon$ equals $m \omega /\left(T_{0} \sigma\right)^{1 / 2}$, where $m$ is the point mass, $T_{0}$ is the tension in the string, $\sigma$ is the mass density of the string, and $\omega$ is the angular frequency of a harmonic wave. For both quantum and classical systems, the transmission coefficient $T\left(=|\tau|^{2}\right)$ and the transmission amplitude $|\tau|$ display resonant behavior: they peak at resonant frequencies. The widths of the resonant peaks obtained by using $T$ and $|\tau|$ are of the same order of magnitude.

${ }^{1}$ C. Cohen-Tannoudji, B. Diu, and F. Laloe, Quantum Mechanics (Wiley, New York, 1977).

${ }^{2}$ A. P. French and E. F. Taylor, Introduction to Quantum Physics (W. W. Norton \& Company, Inc., New York, 1978), pp. 351-354.

${ }^{3}$ E. R. Brown, T. C. G. Sollner, W. D. Goodhue, and C. D. Parker, "Millimeter-band oscillations based on resonant tunneling in a doublebarrier diode at room temperature," Appl. Phys. Lett. 50(2), 83-85(1987). ${ }^{4} \mathrm{H}$. Georgi, The Physics of Waves (Prentice-Hall Englewood Cliffs, NJ, 1993).

${ }^{5}$ S. Parmley et al., "Vibrational properties of a loaded string," Am. J. Phys. 63(6), 547-553(1995).

${ }^{6}$ L. Cohen, Time-Frequency Analysis (Prentice-Hall, Englewood Cliffs, NJ, 1995). 